\title{
Large Scale Structure in an Era of Precision CMB Measurements
}

\author{
Asantha Cooray \\ Theoretical Astrophysics Including Relativity Group, California Institute of \\ Technology, Pasadena, CA 91125, USA
}

\begin{abstract}
The advent of high signal-to-noise cosmic microwave background (CMB) anisotropy experiments now allow detailed studies on the statistics related to temperature fluctuations. The existence of acoustic oscillations in the anisotropy power spectrum is now established with the clear detection of the first, and to a lesser confidence the second and the third, peak. Beyond the acoustic peak structure associated with CMB photon temperature fluctuations, we study the possibility for an observational detection of oscillations in the large scale structure (LSS) matter power spectrum due to baryons. We also suggest a new cosmological test using the angular power spectrum of dark matter halos, or clusters of galaxies detected via wide-field surveys of the large scale structure. The standard rulers of the proposed test involve overall shape of the matter power spectrum and baryon oscillation peaks in projection. The test allows a measurement of the angular diameter distance as a function of redshift, similar to the distance to the last scattering surface from the first acoustic peak in the temperature anisotropy power spectrum. The simultaneous detection of oscillations in both photons and baryons will provide a strong, and a necessary, confirmation of our understanding related to the physics during the recombination era. The proposed studies can be carried out with a combined analysis of CMB data from missions such as the MAP and the large scale structure data from missions such as the DUET.
\end{abstract}

\section{Oscillations in CMB}

The cosmic microwave background (CMB) is now a well known probe of the early universe. The temperature fluctuations in the CMB, especially the so-called acoustic peaks in the angular power spectrum of CMB anisotropies, capture the physics of primordial photon-baryon fluid undergoing oscillations in the potential wells of the dark matter [1]. The associated physics - involving the evolution of a single photon-baryon fluid under Compton scattering and gravity - are both simple and linear, and many aspects of it have been discussed in the literature since the early 1970s [2]. The gravitational redshift contribution at large angular scales [3] and the photon-diffusion damping at small angular scales [4] complete this description.

By now, there are at least five independent detections of the first, and possibly the second and the third, acoustic peak in the anisotropy power spectrum [0.6]. We summarize these results in figure 1. Given the variety of experiments that are either collecting data or reducing data that were recently collected, more detections that extend to higher peaks are soon expected. The NASA's 


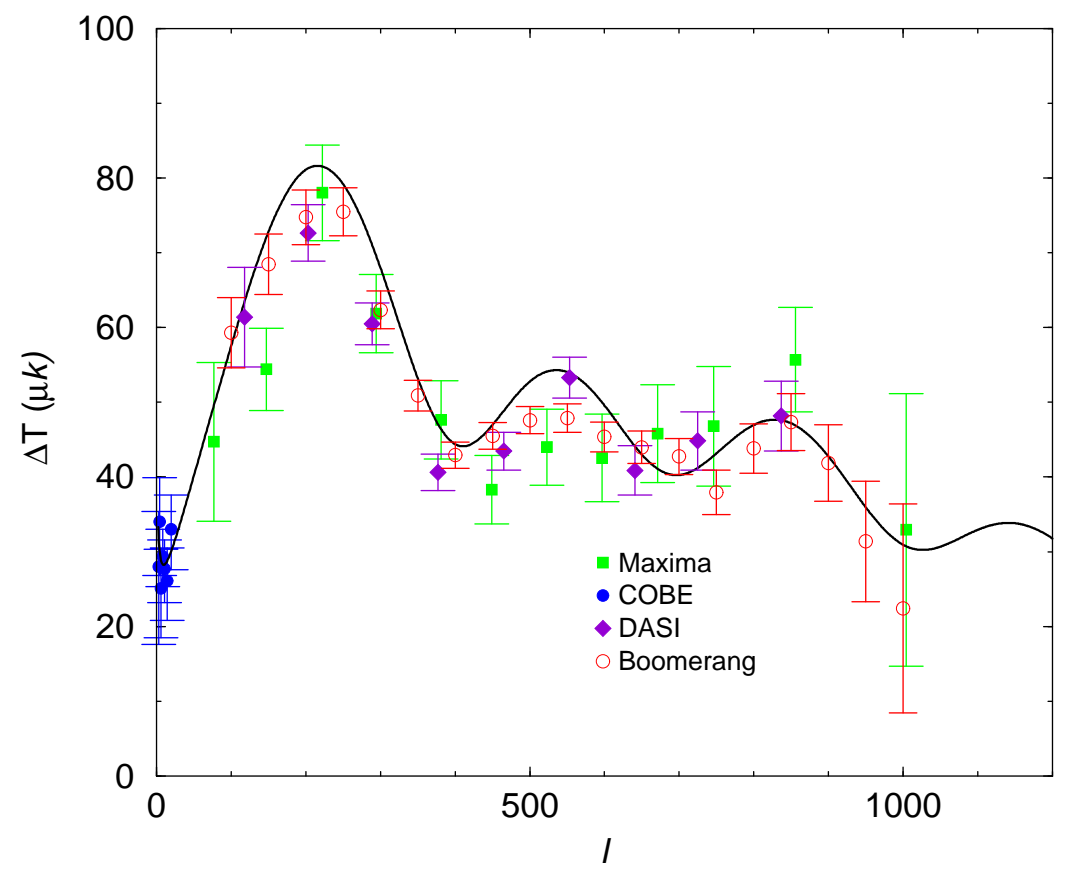

Fig. 1. Temperature fluctuations in the cosmic microwave background as seen by COBE (filled circles; [7]), Boomerang (open circles; 8]), MAXIMA (squares; [9]) and DASI (diamonds; 6]). The solid line shows the theoretical expectation for a cosmology with best-fit parameters for the Boomerang data following 8$]$.

MAP mission 1 is expected to provide a significant detection of the acoustic peak structure out to a multipole of $\sim 1000$ and, in the long term, the ESA's Planck surveyor 2 , will extend this to a multipole of $\sim 2000$ with better frequency coverage and polarization sensitivity. A discussion of these recent results and implications for cosmology are presented in the contribution to these proceedings by A. Melchiorri.

An additional important aspect with respect to $\mathrm{CMB}$ is that in transit to us, photons are affected by the large scale structure along the way. The modifications come from effects related to gravity, such as through frequency shifts associated with the integrated Sachs-Wolfe effect [3], and through effects related to scattering such as the Sunyave-Zel'dovich (SZ) effect [11]. We refer the reader to Ref. [12] for a discussion of these contributions and their importance for understanding the large scale structure via precision CMB measurements.

\footnotetext{
${ }^{1}$ http://map.gsfc.nasa.gov

2 http://astro.estec.esa.nl/Planck/
} 

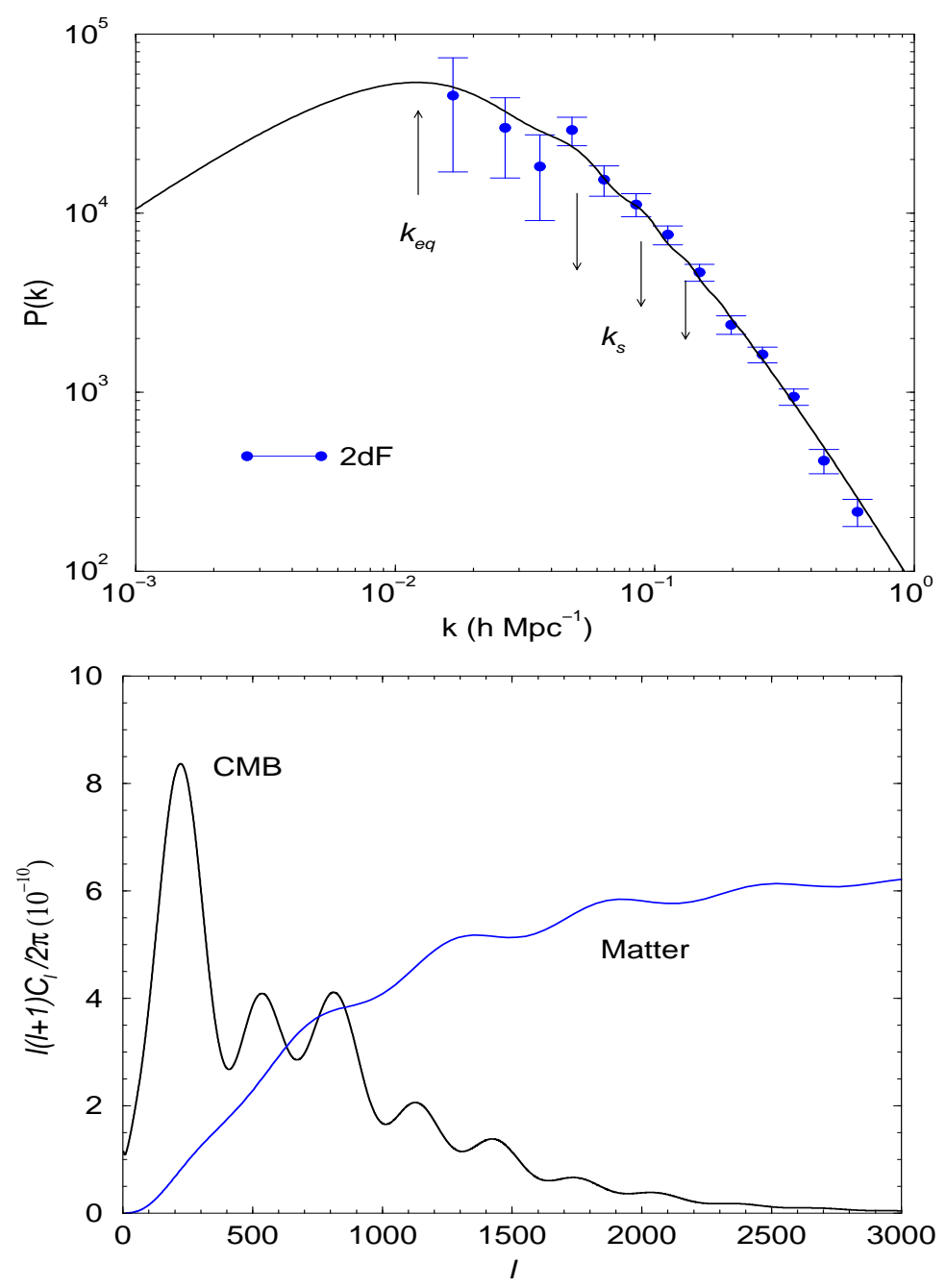

Fig. 2. Top: Large scale structure matter power spectrum. The data are from the analysis of $2 \mathrm{dF}$ redshift survey 10 . We note two important physical scales of interest to the present discussion involving the matter-radiation equality $k_{\text {eq }}$, which produces a turnover in the power spectrum, and the peak location of baryon oscillations corresponding to the projection of the sound horizon at the end of the Compton-drag epoch $k_{\mathrm{s}}$. Bottom: A comparison of the oscillations in CMB due to photons and in matter due to baryons. We project the matter power spectrum at the distance of the last scattering surface following equation (2), and normalize it arbitrarily for illustrative purposes. Note that the acoustic oscillations in the matter power spectrum are out of phase with oscillations in the CMB anisotropy power spectrum.

\section{Oscillations in LSS}

The detection of coherent oscillatory features in the CMB temperature anisotropy power spectrum suggests that if our physical description of them is correct, then, 
the large scale structure at low redshifts should also contain a signature of oscillations. These troughs and peaks are associated with baryons which under went acoustic oscillations with photons as a single photon-baryon fluid prior to recombination?. Unlike oscillations in the angular power spectrum of $\mathrm{CMB}$ anisotropies, oscillations due to baryons in the matter power spectrum of the large scale structure are highly suppressed due to the low baryon content. They are also easily erased at late times due to the non-linear gravitational evolution of density perturbations 114. Compared to amplitudes of oscillations in the radiation, which vary by at least a factor of 2 , baryon oscillations have amplitudes which are significantly lower with variations at the level of $\sim 8 \%$ for currently favored $\Lambda \mathrm{CDM}$ cosmologies. These oscillations have effective widths in Fourier space of order $\Delta k \sim 0.02 \mathrm{~h} \mathrm{Mpc}^{-1}$. As we discuss, these issues put several strong observational constraints for a reliable detection of these oscillations.

The verification on the presence of baryon oscillations in the large scale structure, however, is not impossible. Several attempts have already been made with three-dimensional redshift surveys such as the 2dF Galaxy Redshift Survey and information from measurements related to angular clustering [15], though there is still no significant evidence for the presence of baryon oscillations. The current three-dimensional surveys lack the required volume, with sizes of order $L \sim 2 \pi / \Delta k \sim 300 \mathrm{~h}^{-1} \mathrm{Mpc}$ in all three dimensions, to reliably resolve the oscillations while three dimensional power spectra obtained through an inversion of the angular correlation function should not contain oscillatory features due to the nature of the inversion and the widths of window functions involved, given the expected widths of oscillatory features [16].

While ongoing redshift surveys such as the Sloan Digital Sky Survey (SDSS; 117) allow a strong possibility to detect these oscillations, in future, one can also use projected clustering in Fourier space, such as the angular power spectrum similar to that of CMB anisotropies, to detect baryon oscillations. This is useful given that one does not require precise redshift information but rather spatial information, and estimates of redshift for binning purposes, from wide-field surveys. In addition to galaxy survey data, a number of additional observational efforts are under way or planned to image the large-scale structure of the universe out to redshift of a few. These wide-field surveys typically cover tens to thousands of square degrees on the sky and include the weak gravitational lensing shear observations with instruments such as the SNAP and the Large Aperture Synoptic Survey Telescope, dedicated small angular scale CMB telescopes that will map the SZ effect through instruments such as the South Pole Telescope,

\footnotetext{
3 The oscillations in CMB, due to radiation, and the oscillations in LSS, due to baryons, involve similar physics, though they have subtle differences. These include a phase difference, $\pi / 2$ at small wavenumbers, between the radiation and matter oscillatory peaks, due to a velocity term that source the growing mode of matter perturbations. Also, radiation oscillations project the sound horizon at the recombination while baryon oscillations project the sound horizon at the end of the Compton-drag epoch. In principle, these two epochs can be different, but for all purposes, the horizons are similar for currently favorable $\Lambda \mathrm{CDM}$ cosmological models. We refer the interested reader to discussions in [13,14], and references therein, for further details.
} 


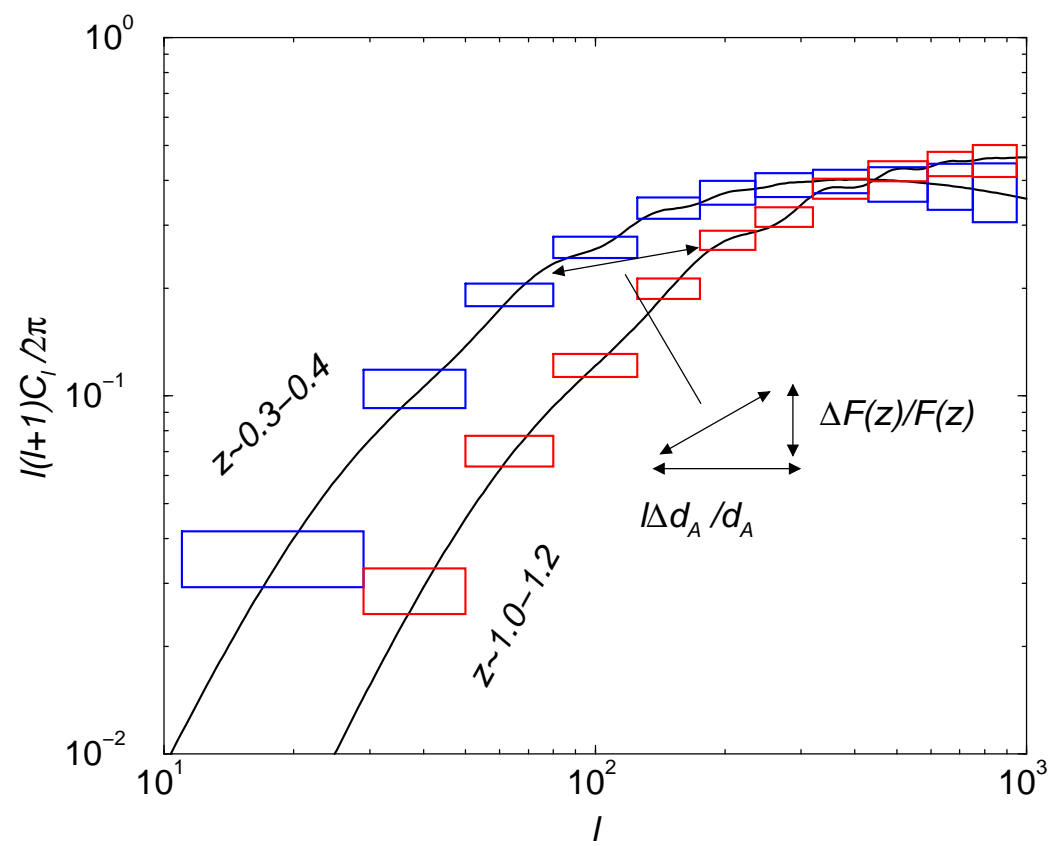

Fig. 3. Angular power spectrum of halos with $M>10^{14} M_{\odot}$ in a wide-field survey in bins of $z=0.3-0.4$ and $1.0-1.2$. The binned errors are $1-\sigma$, and assume a survey of $4000 \mathrm{deg}^{2}$, within reach of upcoming weak lensing and SZ surveys. The angular power spectrum at high redshifts is shifted towards the right proportional to the increase in the comoving angular diameter distance.

and the wide-field X-ray imaging survey using the Dark Universe Explorer Telescope (DUET) mission. The latter was recently proposed to NASA as a Medium Explorer mission.

In addition to their primary science goals, these surveys are expected to produce catalogs of dark matter halos, which in the case of lensing and SZ surveys, such catalogs are expected to be essentially mass selected 18]. In the case of X-ray, using cluster temperature data, one can also construct a mass selected catalog of galaxy clusters. Lensing and other optical surveys are particularly promising in that they will provide photometric redshifts on the member galaxies of a given halo; this will render accurate determination of the halo redshift. Halo number counts as a function of redshift is a well-known cosmological test [19. We can also consider the additional information supplied by the angular clustering of halos, in particular, a possibility to use them for a detection of the baryon oscillations, and to use clustering information for a new cosmological test.

It is well-known that a feature in the angular power spectrum of known physical scale and originating from a known redshift can be used to measure the angular diameter distance between us and this redshift; this has most notably 


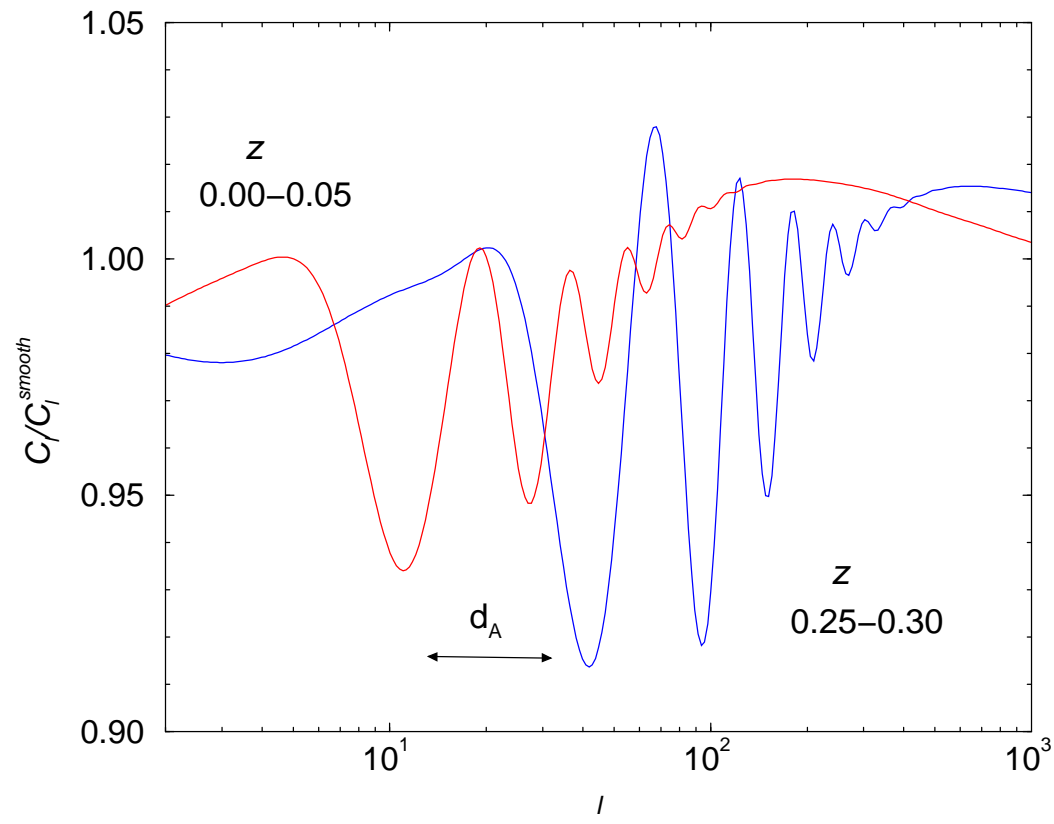

Fig. 4. The normalized power spectrum of halos at $\mathrm{z} \sim 0.05$ and $\mathrm{z} \sim 0.3$ measured in redshift bins of width $\Delta_{z}=0.05$. The shift in oscillations is due to the projection and corresponds to the change in comoving angular diameter distance between the two redshift bins. The increase in amplitude of the oscillations in the higher redshift bin is due to the projection across a smaller width in the comoving angular diameter distance, though the bin width in redshift is same for the two curves.

been applied to the case of CMB anisotropy power spectrum to determine the distance to redshift $z \sim 10^{3}$. Here, one uses the peak location of the first acoustic peak which projects on the multipolar space as $l_{\text {peak }}=k_{*} d_{A}(z=\mathrm{rec})$, where $k_{*}$ is the sound horizon at the recombination and the $d_{A}$ is the associated angular diameter distance in comoving coordinates. Given the location,and a physical model for $k_{*}$, one can constrain cosmology using the variation of $d_{A}$ as a function of cosmological parameters.

We can apply a similar argument to estimate cosmology using the clustering related to the large scale structure. In figure 2, we show the three-dimensional power spectrum and recent measurements from the $2 \mathrm{dF}$ galaxy redshift survey, following the analysis by [10]. In the case of the large scale structure, there are two prominent features in the linear power spectrum associated with the clustering of matter. These are the horizon at the matter-radiation equality

$$
k_{\mathrm{eq}}=\sqrt{2 \Omega_{m} H_{0}^{2}\left(1+z_{\mathrm{eq}}\right)} \propto \Omega_{m} h^{2}
$$

which controls the overall shape of the power spectrum, including a turn over, and the sound horizon at the end of the Compton drag epoch, $k_{\mathrm{s}}\left(\Omega_{m} h^{2}, \Omega_{b} h^{2}\right)$; 
the latter controls the peak location of oscillations due to baryons in the power spectrum. We denote these features in figure 2. Similar to the projection of the sound horizon at the recombination on to the CMB anisotropy power spectrum, the angular, or multipole, locations of features in the large scale power spectrum shift in redshift as $l_{\mathrm{eq}, \mathrm{s}}=k_{\mathrm{eq}, \mathrm{s}} d_{A}\left(z_{i}\right)$. In [20], this allowed us to propose the following test: measure angular power spectrum of projected clustering, $C_{l}^{i}$, in several redshift bins and, using the fact that $l_{\mathrm{eq}, \mathrm{s}}$ scales with $d_{A}\left(z_{i}\right)$, constrain the angular diameter distance as a function of redshift. Unlike the case with CMB, we can use tracers over a wide range in redshift and measure the distance as a function of redshift, or redshift bin. When combined with CMB observations, note that the absolute physical scale of $k_{\text {eq,s }}$ can be directly calibrated as CMB anisotropies provide estimates of parameters such as $\Omega_{m} h^{2}$ and $\Omega_{b} h^{2}$ accurately. When combined with the large scale structure, one can break degeneracies, such as the one between $\Omega_{m}$ and $h$, or given sufficient prior information on $h$ to constrain information related to $\Omega_{m}$ and $\Omega_{\Lambda}$.

To understand the projected clustering, consider the angular power spectrum of a tracer field of large scale structure in a redshift bin. This is simply the projection of the the tracer density power spectrum

$$
C_{l}^{i}=\int d z W_{i}^{2}(z) \frac{H(z)}{d_{A}^{2}(z)} P_{h h}\left(\frac{l}{d_{A}} ; z\right),
$$

where $W_{i}(z)$ is the distribution of tracers in a given redshift bin normalized so that $\int d z W_{i}(z)=1, H(z)$ is the Hubble parameter. For comparison, in the bottom panel of figure 2, we show the projected angular power spectrum of matter at the last scattering surface and the angular power spectrum of CMB anisotropies. Note that the acoustic oscillations associated with photons always show as peaks in the anisotropy power spectrum. There is an overall shift in oscillation phases in the projected angular power spectrum of matter at the last scattering surface when compared to those of CMB anisotropy power spectrum. If we have a tracer of the matter clustering near recombination, say, just after the Compton-drag epoch, we should be able to make a comparison similar to the one suggested in the bottom panel of figure 2 .

For the purpose of this discussion, we assume a tracer of the large scale structure that correspond to mass selected halos, or galaxy clusters. If these halos trace the linear density field,

$$
P_{h h}(k ; z)=\left\langle b_{M}\right\rangle^{2}(z) D^{2}(z) P^{\text {lin }}(k ; 0),
$$

where $\left\langle b_{M}\right\rangle$ is the mass-averaged halo bias parameter, $P^{\text {lin }}(k ; 0)$ is the present day matter power spectrum computed in linear theory, and $D(z)$ is the linear growth function $\delta^{\operatorname{lin}}(k ; z)=D(z) \delta^{\operatorname{lin}}(k ; 0)$. A scale-independent halo bias is commonly assumed in the so-called "halo model" 22 and should be valid at least in the linear regime. Equation (2) then becomes

$$
C_{l}^{i}=\int d z W_{i}^{2}(z) F(z) P^{\operatorname{lin}}\left(\frac{l}{d_{A}^{i}} ; 0\right),
$$



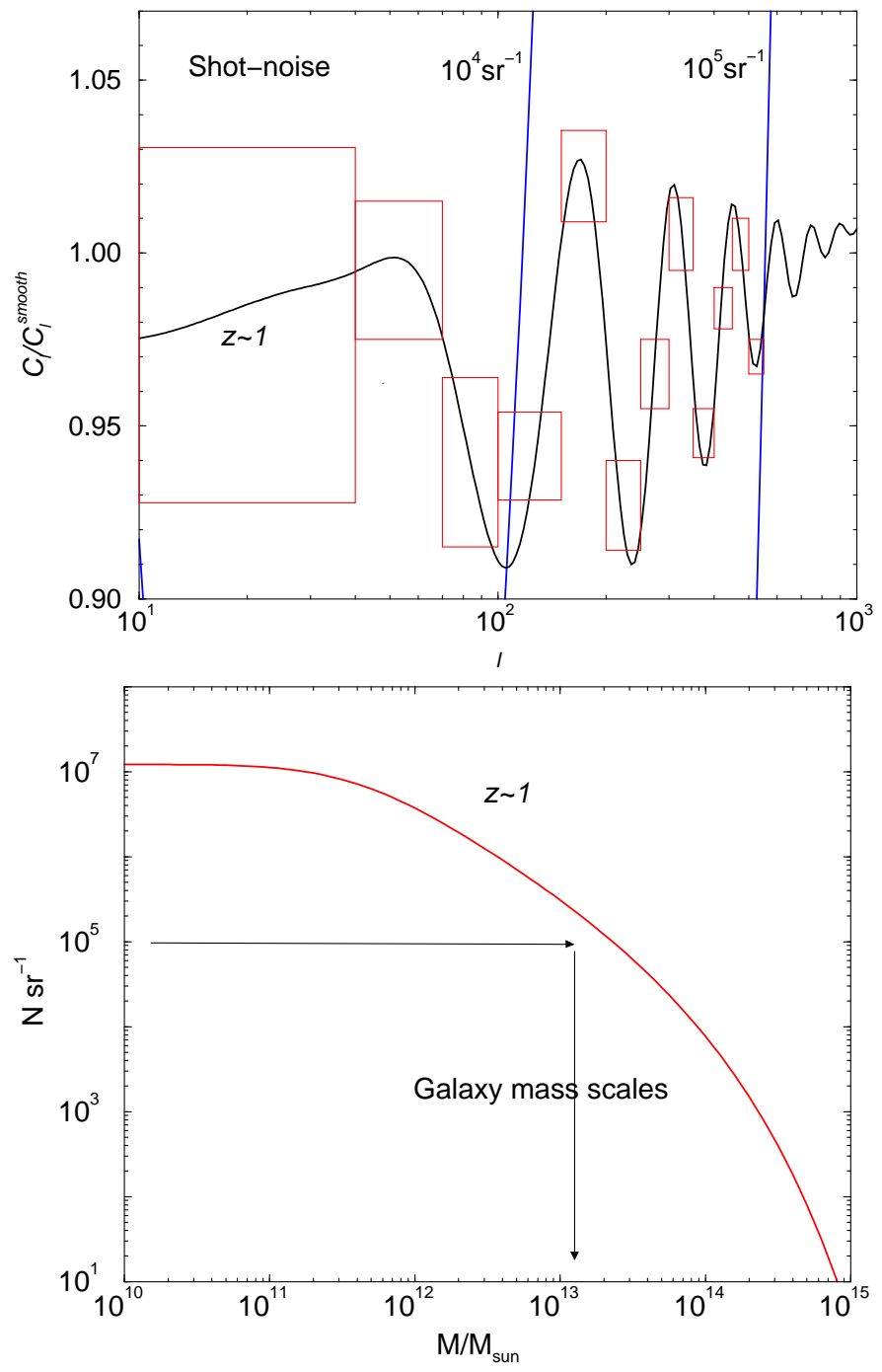

Fig. 5. Expected cosmic variance associated with detection of oscillations around $z$ of 1 , with a redshift bin of 0.1 . The shot-noise contribution due to the finite number of tracers are shown, as a function of surface density of halos, with solid lines. In general, one requires a density of $10^{5} \mathrm{sr}^{-1}$ or more per z-bin for a reliable detection. The bottom panel shows the surface density of halos, as a function of halo mass following the PressSchechter theory [21]. To obtain a surface density of at least $10^{5} \mathrm{sr}^{-1}$, one needs to survey down to mass scales of $10^{13} \mathrm{M}_{\odot}$ at a $z \sim 1$.

$$
F(z)=\frac{H(z)}{d_{A}^{2}(z)} D(z)^{2}\left\langle b_{M}\right\rangle^{2}(z)
$$



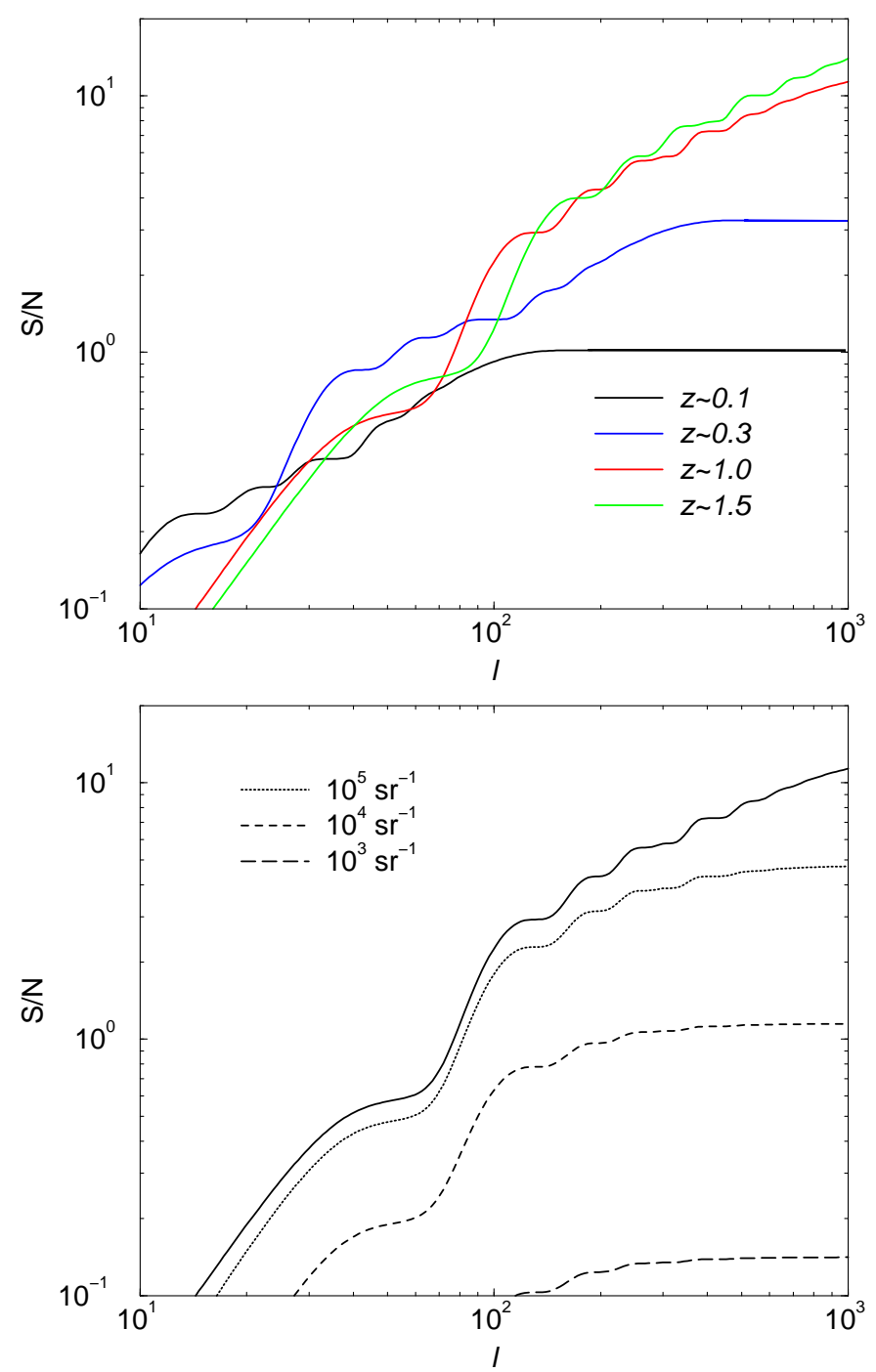

Fig. 6. The cumulative signal-to-noise ratios associated with the detection of baryons oscillations. The top plot illustrates signal-to-noise values as a function of the mean redshift of bins with width 0.1 . The bottom panel shows the effect of finite number counts for the bin at $z \sim 1$. In general, one should expect to detect oscillations with a cumulative signal-to-noise ratio of $\sim 10$.

where the function $F_{i}(z)$, associated with redshift bin $i$, contains information on the halo bias, the growth function, the power spectrum normalization, and terms involved in the 3 -D to $2-\mathrm{D}$ projection (such as a $1 / d_{A}^{2}$ term; see, Eq. (2)). Note that $W_{i}(z)$ comes directly from the observations of the number counts as a function of redshift. 

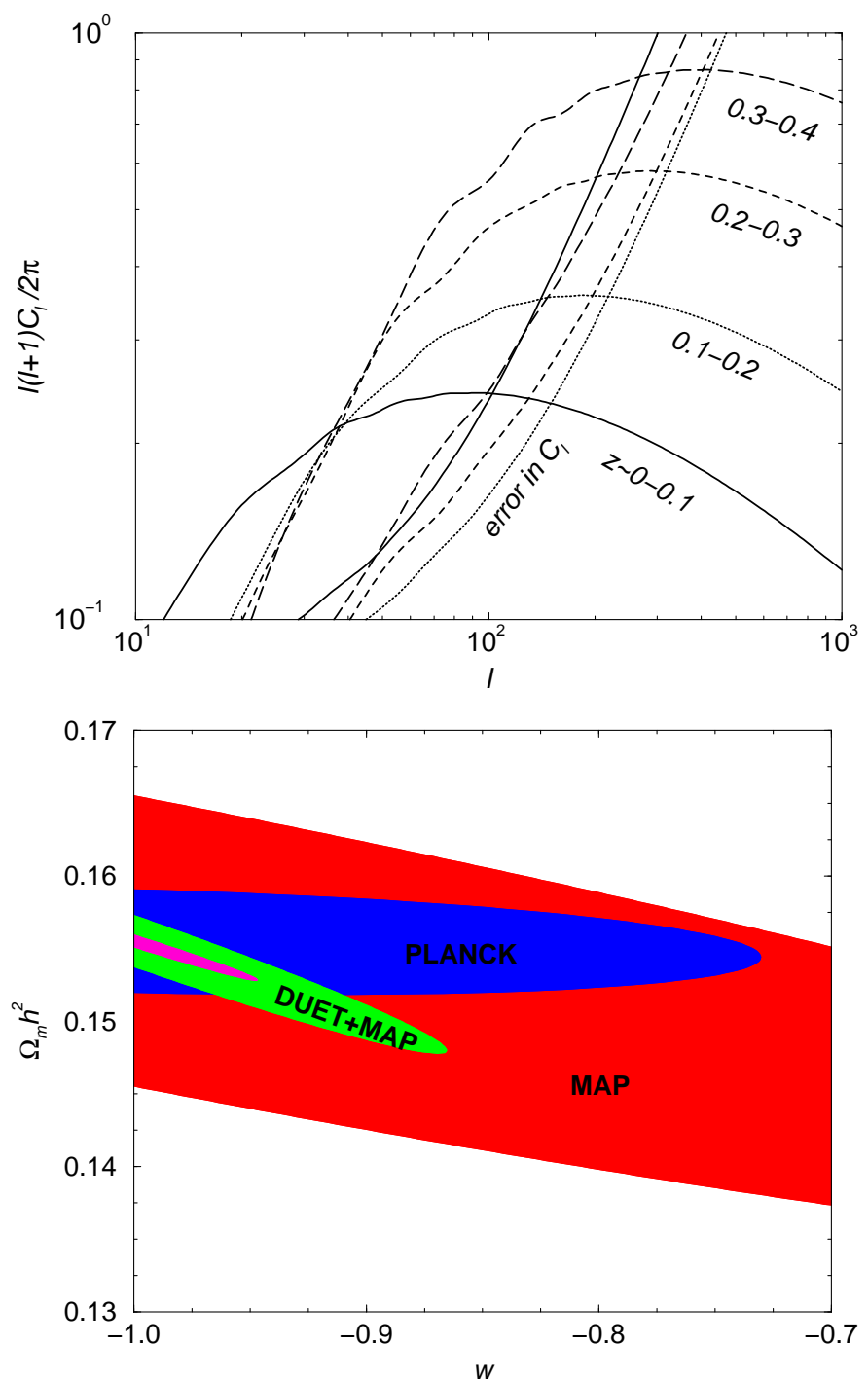

Fig. 7. The expected errors for detection of the clustering signal in catalogs produced by the proposed DUET mission. As shown in the bottom panel, using the overall shape of the angular clustering power spectrum, due to the matter-radiation equality, and the projected locations of baryon oscillations, we can make significant measurements of cosmology. Combined with CMB data, such as the MAP and Planck missions, the DUET will improve errors of certain parameters, such as properties related to the dark energy, by a factor of 5 .

In figure 3, we illustrate the proposed test. The two curves show the halo power spectra in two redshift bins: $0.3<z<0.4$ and $1.0<z<1.2$. The angular power spectrum corresponding to the higher redshift bin is shifted to the right in 
accordance to the ratio of angular diameter distances $\left(\delta l / l \sim \delta d_{A} / d_{A}\right)$. Much of this shift simply reflects the Hubble law, $d_{A} \approx z / H_{0}$. Since the physical size of the two features - the overall shape of the spectrum and the baryon oscillations can be calibrated from the CMB, these measurements can in principle be used to determine the Hubble constant independently of the distance ladder. In figure 4 , we remove the smooth curves and focus only on oscillations. It is clear from this figure that peak locations of oscillations shift in multipole space as a function of the redshift, or more appropriately, the comoving distance.

The ability to measure oscillations crucially depend on both the cosmic variance and the shot-noise associated with the tracer number counts. We illustrate this in figure 5. In the top panel we show the associated cosmic variance errors for an all-sky survey that image two-dimensional clustering at $z \sim 1$. The vertical lines correspond to errors resulting from the finite number of halos. For a reliable detection of the first three baryon oscillations, one needs a survey with a tracer surface density of $10^{5} \mathrm{sr}^{-1}$ and this surface density corresponds to a mass limit of $10^{13} \mathrm{M}_{\odot}$ at this redshift. The cumulative signal-to-noise ratios are illustrated in figure 6. These are the values to distinguish oscillations, which we define for this purpose as any deviation about the mean prediction for clustering in the absence of any baryons.

Note that in addition to the horizontal shift due to the change in angular diameter distance, the power spectra in figure 3 are shifted vertically due to the change in $F(z)$ (Eq. 5). By ignoring the information contained in $F(z)$, the proposed geometric test in [20] is robust against uncertainties in the mass selection, mass function and linear bias. Of course, if these uncertainties are pinned down independently, both $F(z)$ and the halo abundance in $W_{i}(z)$ will help measure the growth rate of structure. In [20], we discussed in detail cosmological information that can be gained from such a test using halos that will be detected in planned SZ and lensing surveys.

We can perform a similar test with cluster catalogs that are expected to be produced with upcoming wide-field X-ray surveys such as the one planned with the proposed DUET mission. The DUET mission plans to map the $\pi$-steradians of the SDSS and the SZ deep fields to be observed with the South Pole Telescope. We show expected errors and improvements in cosmological parameters by combining the DUET clustering information with CMB data in figure The DUET mission allows a detection of baryon oscillations in the projected angular power spectra of galaxy clusters at the few sigma level. This, by itself, is enough to break significant degeneracies associated with cosmological parameter measurements from CMB data. Since the combined test measures cosmology through the angular diameter distance at low redshifts, from clustered halos, and at the last scattering, from the CMB acoustic peak, the test allows one to probe not only the curvature but also relative abundances of various energy densities and properties of the dark energy.

The bottom panel of figure 7 shows errors on $w$, the ratio of pressure to density of the dark energy component, and $\Omega_{m} h^{2}$, from MAP and Planck, and improvements on these errors when these CMB data sets are combined with 
clustering information from the DUET survey. There is at least a factor of 5 improvement in error associated with $w$. Such an improvement clearly demonstrate the need for combined studies involving large scale structure and CMB. We strongly encourage combined approaches such as the one proposed with DUET and CMB data both to understand astrophysics, mainly the presence of baryon oscillations, and cosmology, such as properties of the dark energy.

\section{Acknowledgments}

We thank Zoltan Haiman, Wayne Hu and Dragan Huterer for collaborative work discussed in this article. The author is grateful to the DUET science team for providing him an opportunity to contribute aspects related to a combined CMB and large scale structure data analysis. The author acknowledges support from

the Sherman Fairchild foundation and the Department of Energy and apologizes, before hand, for any missed references.

\section{References}

1. Hu W., Sugiyama N., Silk J. 1997, Nature 386, 37.

2. Peebles, P.J.E. and Yu, J. T. 1970, ApJ, 162, 815; Sunyave, R. A. and Zel'dovich, Ya. B. 1970, Astrop. Space Sci., 7, 3.

3. Sachs, R. K., \& Wolfe, A. M., 1967, ApJ, 147, 73.

4. Silk, J. 1968, ApJ, 151, 459.

5. Miller, A. D. et al., 1999, ApJ, 524, L1; de Bernardis, P. et al., 2000, Nature, 404, 955; Hanany, S. et al., 2000, ApJ, 545, L5.

6. Halverson, N. W. et al., 2001, preprint, astro-ph/0104489.

7. Tegmark, M. 1996, ApJ, 464, 35.

8. Netterfield, C. B. et al. 2001, ApJ in press, astro-ph/0104460.

9. Lee, A. T. et al. 2001, ApJ, 561, L1.

10. Tegmark, M., Hamilton, A.J.S. and Xu, Y. 2001, MNRAS submitted astro$\mathrm{ph} / 0111575$ ).

11. Sunyaev, R.A. \& Zel'dovich, Ya. B. 1980, MNRAS, 190, 413.

12. Cooray, A. 2002, in 2001 Coral Gables conference on cosmology and particle physics, Eds. B. Kursunoglu \& A. Perlmutter, American Institute of Physics Conference Proceedings astro-ph/0203048).

13. Hu, W. and Sugiyama, N. 1996, ApJ, 471, 542.

14. Meiksin, A. White, M. and Peacock, J. A. 1996, MNRAS, 304, 851.

15. Percival, W. J. et al. 2001, MNRAS, 327, 1297; Miller, C. J., Nichol, R. C. and Batuski, D. J. 2001, Sci., 292, 2302.

16. Cooray, A. et al. 2002, in preparation.

17. York, D. G. et al. 2000, AJ, 120, 1579.

18. Wittman, D., Tyson, J. A., Margoniner, V. E., Cohen, J. G., Dell'Antonio, I. P. 2001, ApJ, 557, L89; Holder, G. P., Mohr, J. J., Carlstrom, et al. 2000, ApJ, 544, 629.

19. Haiman, Z., Mohr, J. J., Holder, G. P. 2001, 553, 545.

20. Cooray, A., Hu, W., Huterer, D. and Joffre, M. 2001, ApJ, 557, L7.

21. Press, W. H., Schechter, P. 1974, ApJ, 187, 425

22. Cooray, A. \& Sheth, R. 2002, Physics Reports, submitted. 